\title{
Tensile Bond Strength of Er, Cr: YSGG Laser-irradiated Human Dentin to Composite Inlays with Two Resin Cements
}

\author{
Wan-Yu TSENG ${ }^{1}$, Min-Huey CHEN ${ }^{1}$, Hui-Hsin LU², Chii-Wann LIN ${ }^{3}$, Tseng-Ting HSIEH ${ }^{1}$, Chun-Hao CHEN ${ }^{1}$, \\ Juin-Yih $\mathrm{LAI}^{4}$ and Bor-Shiunn LEE ${ }^{1}$ \\ ${ }^{1}$ Graduate Institute of Clinical Dentistry, College of Medicine, National Taiwan University and National Taiwan University \\ Hospital, No. 1, Chang-Te Street, Taipei 10016, Taiwan \\ ${ }^{2}$ Department of Electrical Engineering, National Taiwan University, No. 1, Chang-Te Street, Taipei 10016, Taiwan \\ ${ }^{3}$ Institute of Biomedical Engineering, College of Medicine and College of Engineering, National Taiwan University, No. 1, \\ Chang-Te Street, Taipei 10016, Taiwan \\ ${ }^{4}$ R\&D Center for Membrane Technology, Department of Chemical Engineering, Chung Yuan University, Chung Li 32023, \\ Taiwan \\ Corresponding author, Bor-Shiunn LEE; E-mail: shiunn@ha.mc.ntu.edu.tw
}

Received October 30, 2006/Accepted May 30, 2007

\begin{abstract}
The purpose of this study was to investigate the tensile bond strength of composite inlays to human dentin with two different cavity preparation tools (bur versus Er, Cr: YSGG laser) and with two different bonding procedures (total-etch: Variolink II versus self-etch: Maxcem). The specimens were divided into four groups: bur-cut/Variolink II, bur-cut/Maxcem, laser-ablated/Variolink II, and laser-ablated/Maxcem. The following characteristics were then investigated: morphological change, dentin-resin cement interface, surface roughness, and tensile bond strength. Results demonstrated that the tensile bond strengths of the four groups were 19.11 $\pm 5.88 \mathrm{MPa}, 8.54 \pm 2.38 \mathrm{MPa}, 13.72 \pm 3.43 \mathrm{MPa}$, and $12.11 \pm 3.71 \mathrm{MPa}$, respectively. We concluded that Variolink II provided higher tensile bond strength to composite inlays than Maxcem. On the other hand, the bond strength of Variolink II with dentin cavity prepared by Er, Cr: YSGG laser was statistically lower than that prepared by bur.
\end{abstract}

Keywords: Er, Cr: YSGG laser, Tensile bond strength, Resin cement

\section{INTRODUCTION}

For several decades, dentists have been using conventional mechanical cutting and drilling systems to remove diseased dental hard tissues and to prepare cavities for restoration. This process is efficient but uncomfortable as the vibrations and sharp noises make patients anxious. Since lasers were introduced for in vitro caries removal ${ }^{1,2)}$, their advantages of decreased discomfort and reduced pain have also led numerous researchers to investigate other potential applications of laser in dentistry.

Unfortunately, ruby, argon, $\mathrm{CO}_{2}$, and $\mathrm{Nd}$ : YAG lasers have been reported by many researchers to produce major thermal effects on dentin, including melting, carbonization, the creation of fissures and cracks in the surrounding tissues, as well as an increase in pulpal temperature. With the discovery of two new laser types, Er: YAG and Er, Cr: YSGG lasers, which were approved by the Food and Drug Administration (FDA), dental hard tissues can now be removed without causing damage.

The Er, Cr: YSGG laser emits a wavelength of 2,780 $\mathrm{nm}$. Using a pulsed-beam system and fiber delivery, it has proved to be a valuable tool for ablating enamel and dentin. It has been studied for Class V cavity preparations and caries removal, and was shown to produce irregular, rugged, and stripped surfaces on dentin, similar to the morphological changes observed in Er: YAG laserirradiated dentin ${ }^{3,4)}$.

Compared with conventional mechanical drilling systems, laser preparation produced a cavity floor with $\mathrm{Ca} / \mathrm{P}$ ratio and Knoop hardness number similar to that produced by bur cutting method ${ }^{5}$. In addition, Er, Cr: YSGG laser preparation was capable of causing significant reductions in Escherichia coli and Enterococcus faecalis ${ }^{6}$. When Er, Cr: YSGG laser preparation was supplemented with acid etching, there were no significant differences in the microleakage of composite fillings in Class II cavities between laser and classical bur preparations ${ }^{7}$. Moreover, pulpal temperature increased by only $2^{\circ} \mathrm{C}$ when dentin was irradiated by Er, Cr: YSGG laser ${ }^{8}$, and no pulpal inflammatory responses could be identified either immediately or 30 days after laser preparation". Furthermore, Er, Cr: YSGG laser could be utilized as an etching tool. A study on the microtensile bond strength of porcelain veneers bonded to tooth surface treated with either Er, Cr: YSGG laser or 37\% orthophosphoric acid was reported to have similar values ${ }^{10}$.

On the other hand, conventional resin cements use etchant, primer or adhesive in the process of bonding tooth-colored inlays to prepared cavities. Bonding effectiveness might thus be compromised due to the multi-step application technique ${ }^{11}$. Recently, a self-etch resin cement combining all the three 
components into a tube has been introduced (Maxcem, Kerr Sybron Dental Specialties, Orange, CA, USA). Without the time-consuming procedure of surface pretreatment, the self-etch resin cement has greatly shortened the clinical chairtime.

Although the bond strength of Er: YAG laserirradiated dentin has been investigated ${ }^{12)}$, little effort has been made on comparing the quality of Er, Cr: YSGG laser-irradiated dentin surface for composite inlays with either etch-and-rinse or self-etch resin cement. Thus, the primary aim of this study was to investigate the tensile bond strengths of bur-cut and Er, Cr: YSGG laser-ablated human dentin to composite inlays with either etch-and-rinse or self-etch resin cement and to analyze the resin cement-dentin interface.

\section{MATERIALS AND METHODS}

\section{Materials}

Two types of resin cement — self-etch, self-adhesive resin cement, Maxcem (Kerr Co., Orange, CA, USA), and dual- and light-curing resin dental luting material, Variolink II (Ivoclar Vivadent, Schaan, Liechtenstein) — were used in this study. The composite inlay was made of heat- and pressureprocessed polymer-glass dual-cure restorative, belleGlass HP (Kerr/Sybron, Orange, CA, USA). Table 1 lists the compositions of these materials.

\section{Tooth specimens}

Extracted human third permanent molars from 16to 40-year-old individuals were used in this study. They were obtained with informed consent of the donors. Collection of human teeth was performed under the permission of the Ethical Committee of National Taiwan University Hospital (Case No. 9561702045). Crowns with caries, restorations, or fractures were discarded. Any remaining soft tissue was thoroughly removed from the tooth surface with a dental scaler (Sonicflex 2000, KaVo Co., Biberbach, Germany) under running water. All teeth were then stored in $4^{\circ} \mathrm{C}$ distilled water containing $0.2 \%$ thymol to inhibit microbial growth until use. To minimize deterioration, the storage medium was changed every week and all preserved specimens were used for tensile bond test within one month.

While fully hydrated, each molar was first cut just below the occlusal pit and fissure, perpendicular to the long axis of the tooth by means of a lowspeed diamond wafering blade (Isomet, Buehler Ltd., Lake Bluff, IL, USA). Next, the crown dentin disk with a thickness of $2.5 \mathrm{~mm}$ was obtained by a second cut parallel to the first one. The enamel of each specimen was removed with a plain-cut tungsten carbide fissure bur at high speed under continuous water spray. Then, the dentin specimens were wet-polished with 600-grit silicon carbide abrasive paper followed by sonic vibration in distilled water for 30 seconds to create uniform flat surfaces, as well as to remove any superficial debris created during cutting and polishing procedures. These dentin disks were fabricated for analysis of surface morphological changes and for resin cement-dentin interface observation after being bur-cut or Er, Cr: YSGG laserablated.

As for the tensile bond test, specimen preparation procedure was the same except that the second

Table 1 Materials used in this study

Scientific name/Product name (manufacturer)/Lot General composition
number

Self-etch, self-adhesive resin cement/Maxcem (Kerr Co., Orange, CA, USA)/444924

Dual- and light-curing resin cement/Variolink II (Ivoclar Vivadent, Schann, Liechenstein)/H06641

Heat- and pressure-processed polymer-glass dualcure restorative/belleGlass HP

(Kerr Sybron, Orange, CA, USA)/409999
$66 \%$ filler by weight, with an average filler particle size of 3.6 microns; $20-35 \%$ uncured methacrylate ester monomers

Excite DSC: HEMA, dimethacrylates, phosphoric acid acrylate, silicon dioxide, initiators and stabilizers in an alcohol solution Monobond-S: 3-methacryloxypropyl-trimethoxysilane, waterethanol solution

Paste A: Bis-GMA, urethane dimethacrylate, TEGDMA, inorganic filler, ytterbium trifluoride, initiator, stabilizer Paste B: Bis-GMA, urethane dimethacrylate, TEGDMA, inorganic filler, ytterbium trifluoride, benzoyl peroxide, stabilizer

Methacrylate ester monomer/BisGMA/TEGDMA, particulate composite (78\% filler: Ba-Silicate, $\mathrm{SiO}_{2}$ ) 
cut was not performed.

\section{Er, Cr: YSGG laser irradiation settings}

The laser system used in this study was Er, Cr: YSGG laser (Millennium ${ }^{\mathrm{TM}}$ II, Biolase Technology Inc., San Clemente, CA, USA). It emitted a wavelength of $2,780 \mathrm{~nm}$ with a repetition rate of $20 \mathrm{~Hz}$ and a beam spot size of $0.442 \mathrm{~mm}^{2}$. Output power varied from 0 to $6 \mathrm{~W}$. Laser beam was used in a non-contact mode within $1-2 \mathrm{~mm}$ from the target area, and the tip with a fiber of $0.6 \mathrm{~mm}$ diameter was held perpendicular to the lased surface during irradiation. Cavities were prepared with a focused mode and water spray cooling. Energy parameters were $3.5 \mathrm{~W}$ and $20 \mathrm{~Hz}$ with water spray (air pressure level: $80 \%$; water pressure level: maximum level), which were determined in our pilot study to yield better efficiency, minimal thermally induced changes, and favorable surface characteristics.

Specimen preparation for surface morphology observation

Five crown dentin disks were employed to observe the changes in surface morphology. Each disk was equally divided into three areas to receive three different treatments (Groups 1-3). In Group 1, the disks were fixed with utility wax and a cavity was prepared on the allocated surface area in each disk with a high-speed \#411 cylinder diamond bur rotating at 350,000 rpm under air-water spray. The cavity was of $2 \times 2 \mathrm{~mm}^{2}$ size and $1 \mathrm{~mm}$ deep. Then, the cavity was etched with $35 \%$ phosphoric acid gel (Ultra-Etch, Ultradent Products, South Jordan, UT, USA) with an applicator sponge for 30 seconds and rinsed with an air-water spray for 20 seconds. In Group 2, cavities of the same dimensions as Group 1 were prepared using the Er, Cr: YSGG laser at an energy setting of $3.5 \mathrm{~W}$ and $20 \mathrm{~Hz}$ with water spray, but without acid etching. Group 3 received the same treatment as Group 2, but with acid etching.

Specimen preparation for resin cement-dentin interface observation

Another five crown dentin disks were prepared for composite inlay restorations and for resin cementdentin interface observation. Each dentin disk was equally divided into two areas for receiving bur or laser preparation (Groups 4 and 5). Groups 4 and 5 were equally subdivided into two areas for receiving self-etch adhesive resin cement (Maxcem) or total-etch adhesive resin cement (Variolink II) (Table 1). Dimensions of cavities prepared for Groups 4 and 5 were the same as those of Groups 1-3.

After cavity preparation, silicone impression material (Rapid liner, Coltene/Whaledent Inc., Mahwah, NJ, USA) was mixed according to manufa cturer's instructions to make an impression of the cavity. After 30 minutes, type IV dental stone (Durone, Dentsply-DeTrey, Konstanz, Germany) was poured into each mold and allowed to set in a plastic storage box. The stone casts were then separated from the impressions after one hour.

A disposable swab was used to paint a thin layer of separator on the dies and any part of the model that may come in contact with the belleGlass HP composite material. Subsequently, Opaceous Dentin was applied onto the preparation floor. Each increment of composite resin was well condensed to fill the cavity and light-cured with a light-emitting diode (LED) light curing unit (LEDemetron II, SDS Kerr, Danbury, CT, USA) for 40 seconds. Energy output was $750 \mathrm{~mW} / \mathrm{cm}^{2}$ as measured with a power meter (Cure Rite, Dentsply Caulk, Milford, DE, USA). Finally, each die was inserted into the belleGlass HP curing unit chamber for 20 minutes under a specific temperature $\left(135^{\circ} \mathrm{C}\right)$ and pressure $(0.41 \mathrm{MPa})$ within nitrogen environment to reach final cure.

Before cementation with Maxcem or Variolink II, the inner surfaces of manufactured composite inlays were sandblasted with $50-\mu \mathrm{m} \mathrm{Al}_{2} \mathrm{O}_{3}$ particles at an air pressure of $0.25 \mathrm{MPa}$ (PrepStart, PowerPlus Basic Quattro IS, Danville Engineering, San Ramon, CA, USA) for 13 seconds from a distance of $10 \mathrm{~mm}$. Samples were then ultrasonically cleaned in $70 \%$ isopropyl alcohol for three minutes.

Maxcem was a self-etch, self-adhesive resin cement that combined the etchant, primer, and adhesive resin into one material. Before placement of Maxcem, the prepared cavity was rinsed thoroughly followed by air-dry if the remaining water on the surface was not dried completely. Subsequently, the cement was dispensed into the cavity using the mixing tip on dual syringe cartridge. The inlay restoration was properly seated, and all excess cement was removed with an explorer. Finally, the surface was light-cured by LEDemetron II unit for 40 seconds.

For cementation with Variolink II, the cavity was etched with $35 \%$ phosphoric acid gel. The inner surfaces of the inlay restoration were also silanized with Monobond-S for 60 seconds and then dried with water-free air. The dentin cavity and the inner surfaces of the inlay restoration were then saturated with a generous amount of Excite DSC for $10 \mathrm{sec}-$ onds. Subsequently, base paste and catalyst paste in a 1:1 ratio were mixed on a mixing pad with a spatula followed by cementation of inlay. All excess cement was removed, and the surface was light-cured by LEDemetron II unit for 40 seconds.

After storing in distilled water for 24 hours $^{13)}$, these dentin disks were split perpendicular to the dentin-resin cement-inlay interfaces with a straight unibevel end chisel and a dental hammer. Sectioned specimens were demineralized in $6 \mathrm{~N}$ hydrochloric acid (HCl) (Wako Pure Chemical Industries Ltd., 
Osaka, Japan) for one minute, rinsed in distilled water, deproteinized in $1 \%$ sodium hypochlorite solution ( $\mathrm{NaOCl}$ ) (Wako Pure Chemical Industries Ltd., Osaka, Japan) for 10 minutes, and finally rinsed with distilled water again.

\section{Specimen preparation for tensile bond test}

Extracted human third permanent molars were randomly divided into four groups (Groups 6-9: bur-cut/Variolink II, bur-cut/Maxcem, laserablated/Variolink II, and laser-ablated/Maxcem) to receive the same surface treatments as Groups 4 and 5. Each group consisted of 10 specimens. The specimens for tensile bond test were prepared according to a previously published method ${ }^{14)}$. Each trimmed specimen was fixed to a custom-made metal jig using cyanoacrylate and debonded in tension with a universal testing machine (Instron Corp., Canton, MA, USA) at a crosshead speed of $0.5 \mathrm{~mm} /$ minute. The forces to dislodge the bonds between dentin and composite inlays were recorded by the load cell and computer software (Merlin Software Suite, Instron Corp., Canton, MA, USA). Dimensions of each side of the debonded specimens were measured using a digital micrometer (Mitutoyo Co., Tokyo, Japan), and tensile bond strength $(\mathrm{MPa})$ was calculated for each specimen.

Fracture types at the dentin-resin cement-inlay interfaces were analyzed using a digital stereoscopic microscope (Leica MZ8, Heebrugg, Switzerland) at $\times 8-25$ magnification. In addition, fractured surfaces of the debonded specimens were examined by SEM. The following classification was employed to determine the fracture mode: Type 1 - Complete adhesive failure between resin cement and dentin; Type 2 Partial adhesive failure between resin cement and dentin, and partial adhesive failure between resin cement and composite inlay; Type 3 - Complete adhesive failure between resin cement and composite inlay.

\section{Scanning electron microscopy (SEM) evaluation}

The morphological changes after treatment with bur cut or laser ablation, as well as the resin cementdentin interface, were observed under a scanning electron microscope. All specimens were immersed in $2.5 \%$ cold glutaraldehyde in $0.1 \mathrm{~mol} / \mathrm{L}$ cacodylate buffer at $\mathrm{pH} 7.4$ for eight hours. They were then serially dehydrated in graded ethanol solutions (50\%, $60 \%, 70 \%, 80 \%, 90 \%, 95 \%$, and $100 \%$ ethanol) at 45-minute intervals, critical point-dried by $\mathrm{CO}_{2}$, mounted on aluminum stubs, sputter-coated with $\sim 20 \mathrm{~nm}$ of gold-palladium alloy, and finally examined by a Hitachi SEM (Model S-800, Tokyo, Japan) at an accelerating voltage of $15 \mathrm{kV}$.
Atomic force microscopy (AFM) evaluation

To evaluate the surface roughness of specimens after different treatments, three crown dentin disks were prepared via the aforementioned methods. Each disk was equally divided into four areas to receive diamond bur preparation plus acid etching, diamond bur preparation only, laser preparation plus acid etching, and laser preparation only. Laser energy settings and acid etching procedure were the same as those for surface morphology analysis. All imaging experiments were carried out with an AFM (NSCRIPTOR $^{\mathrm{TM}}$, NanoInk Inc., Chicago, IL, USA) and conventional cantilevers (force constant $=0.10 \mathrm{~N} / \mathrm{m}$, $\mathrm{Si}_{3} \mathrm{~N}_{4}$ ). Five squares of each area of $50 \mu \mathrm{m}$ length were randomly selected for scanning with AFM, and the average roughness value was calculated.

\section{Statistical analysis}

Surface roughness and bond strength of each group were analyzed with one-way ANOVA and Tukey's test $(\mathbf{a}=0.05)$ to determine whether there were any significant differences among the groups.

\section{RESULTS}

Morphological analysis of prepared dentin surfaces The morphology of the cavity floor prepared by a high-speed diamond bur and acid-etched with phosphoric acid is shown in Fig. 1(a). Enlarged dentinal tubule orifices were noted due to loss of mineral phase within peritubular and intertubular dentin. No dentin debris or smear layer was observed and some fibrous structures were noted inside the dentinal tubules. The dentin surface irradiated by Er, Cr: YSGG laser showed a scaly, irregular, and rugged appearance (Fig. 1(b)). There was also no smear layer or debris, and dentinal tubule orifices were open without widening. It also showed peritubular dentin protruding from the surrounding intertubular dentin. Additionally, some cracks could be observed. In the laser-ablated dentin surface etched by $35 \%$ phosphoric acid, enlarged dentinal tubule orifices without smear layer were found (Fig. 1(c)). Protrusion of peritubular dentin from intertubular dentin was not noted.

Morphological analysis of dentin-resin cement interface

When the cavity was prepared by a high-speed diamond bur and cemented with Variolink II, numerous resin tags with length of about $50 \mu \mathrm{m}$ appeared in an orderly fashion at the dentin-resin cement interface (Fig. 2(a)). They aggregated in a bulbshaped configuration (arrowhead) and then diverted into dentinal tubules. When the cavity was prepared by a high-speed diamond bur and cemented with Maxcem, no resin tags were found in the 

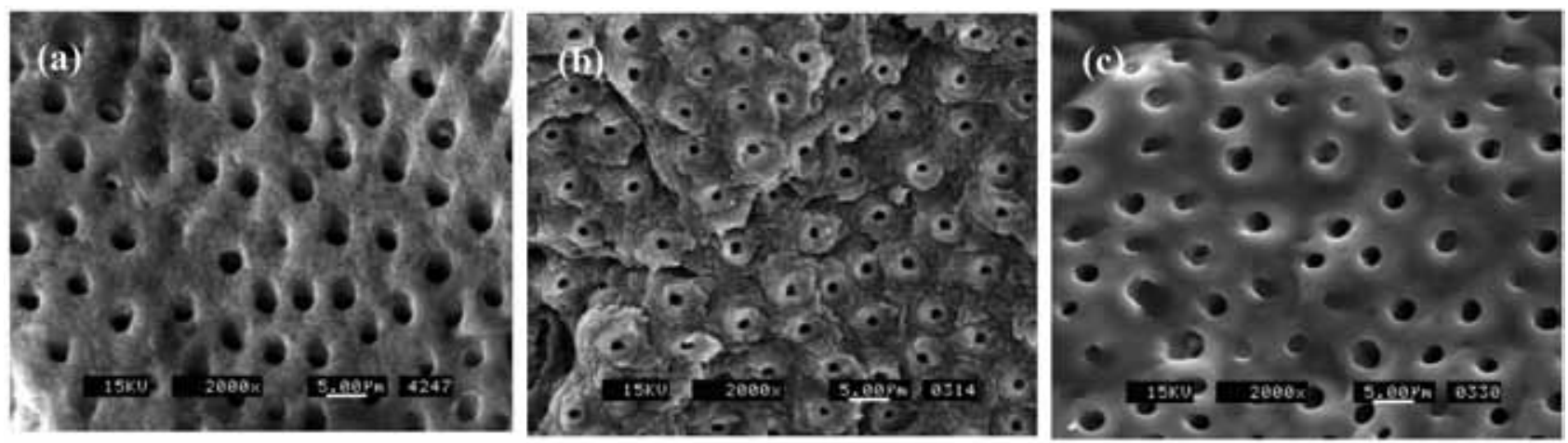

Fig. 1 (a) Microstructure of a cavity floor prepared by a high-speed diamond bur and acid-etched with phosphoric acid showed enlarged dentinal tubule orifices, which were attributed to loss of mineral phase within peritubular and intertubular dentin. (b) Prepared dentin lased by Er, Cr: YSGG laser revealed a scaly, irregular, and rugged appearance. Both dentin surfaces were free of smear layer or debris. (c) When laser-ablated dentin surface was etched by $35 \%$ phosphoric acid, enlarged dentinal tubule orifices without smear layer were noted. No protrusion of peritubular dentin from intertubular dentin was found.
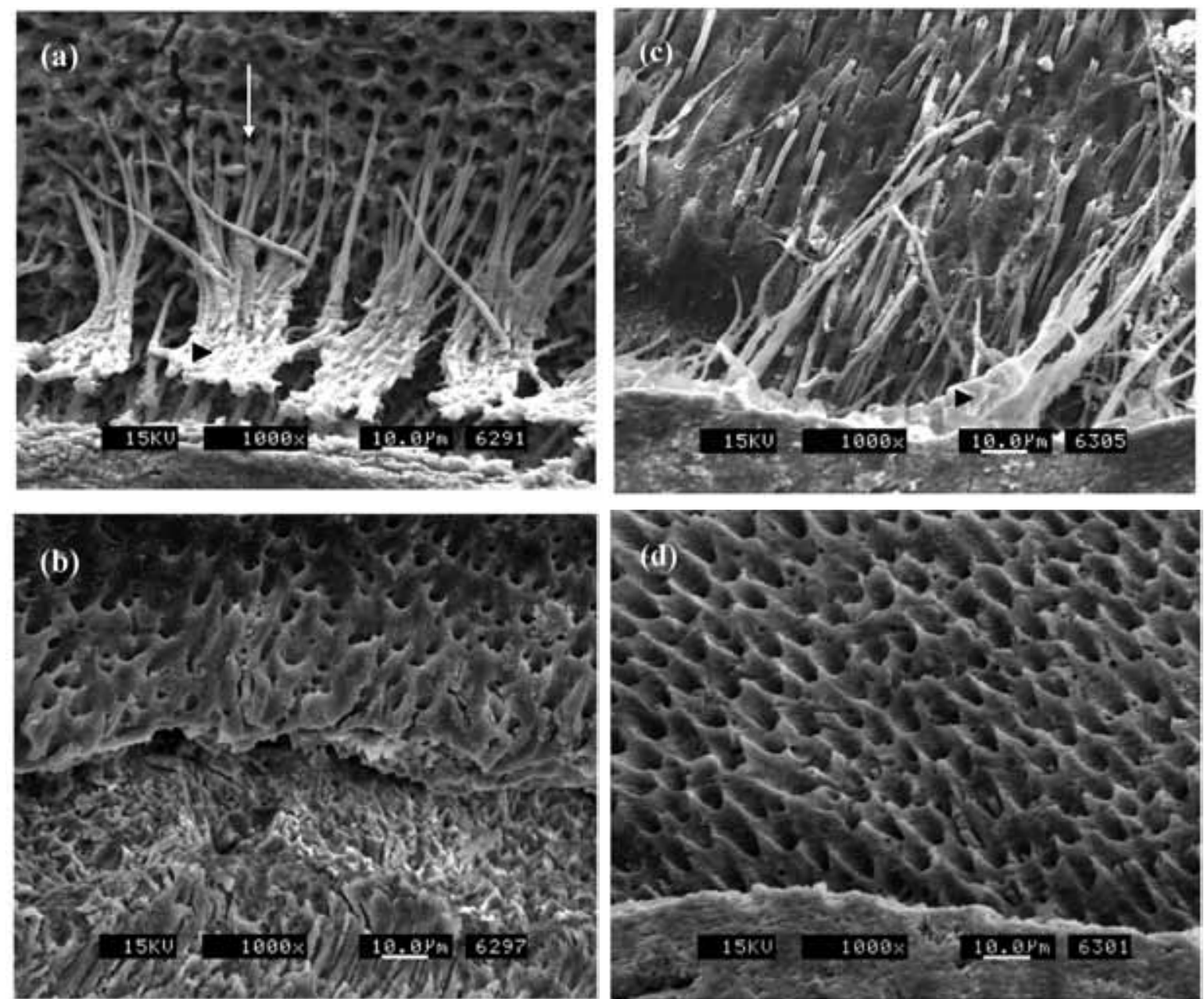

Fig. 2 (a) Numerous resin tags with length of about $50 \mu \mathrm{m}$ (arrow) could be found at the dentin-resin cement interface after receiving bur-cut/Variolink II treatment. Hybrid layer formation (arrowhead) and resin tags diverted into dentinal tubules could be found. (b) No resin tags were found in the microstructure receiving bur-cut/Maxcem treatment. (c) Arrangement of resin tags was irregular and hybrid layer was not well formed (arrowhead) when cavity was prepared by Er, Cr: YSGG laser and cemented with Variolink II. (d) No resin tags existed at the dentin-resin cement interface when cavity was prepared by Er, Cr: YSGG laser and cemented with Maxcem. 


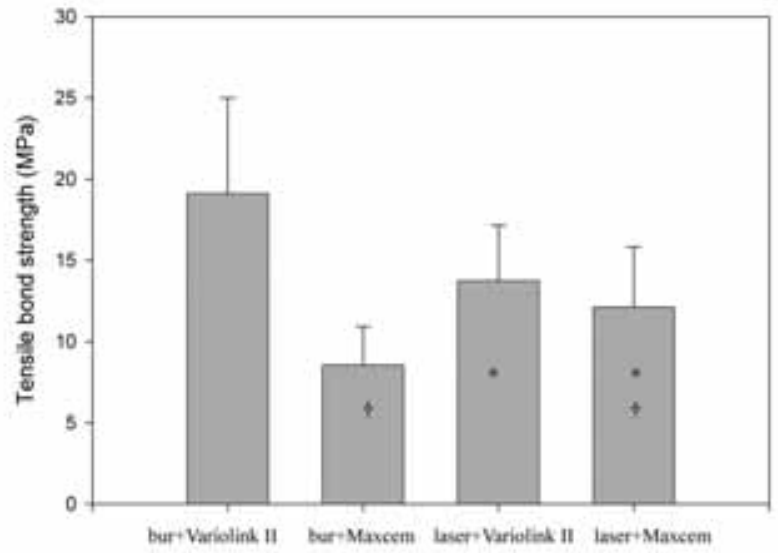

Fig. 3 Means and standard deviations of tensile bond strengths of bur-cut/Variolink II, burcut/Maxcem, laser-ablated/Variolink II, and laser-ablated/Maxcem were $19.11 \pm 5.88 \mathrm{MPa}$, $8.54 \pm 2.38 \mathrm{MPa}, 13.72 \pm 3.43 \mathrm{MPa}$, and $12.11 \pm$ $3.71 \mathrm{MPa}$, respectively. Columns labeled with the same notation (*or $\dagger$ ) indicated that they had no statistically significant differences $(n=$ 10).
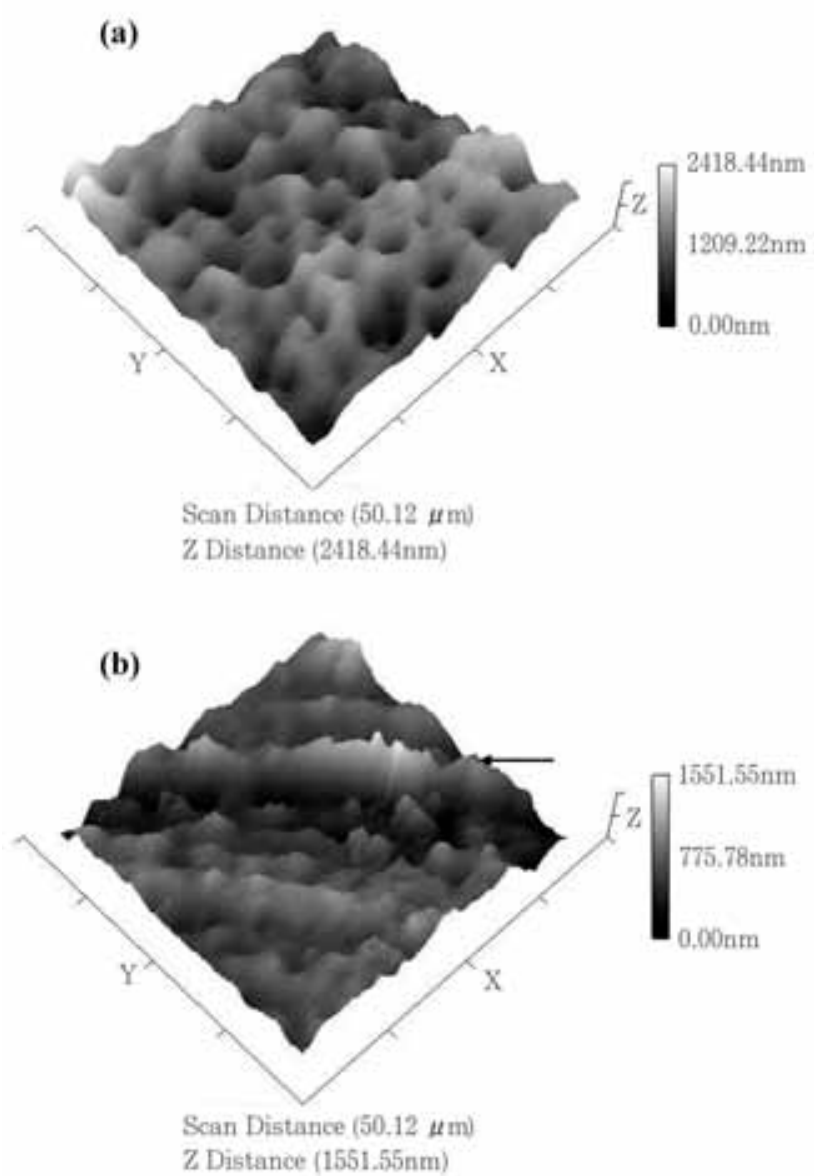

microstructure of dentin-resin cement interface (Fig. 2(b)). Figure 2(c) shows the dentin-resin cement interface of the cavity prepared by Er, Cr: YSGG laser plus cementation with Variolink II. Although some resin tags were observed, their arrangement was irregular and the bulb-shaped configuration was not prominent as in Fig. 2(a). There were also no resin tags at the dentin-resin cement interface when

Table 2 Fracture types by digital stereoscopic microscope and SEM

\begin{tabular}{lccc}
\hline & Type 1 & Type 2 & Type 3 \\
\hline Bur-cut/Variolink II & 7 & 1 & 2 \\
Bur-cut/Maxcem & 10 & 0 & 0 \\
Laser-ablated/Variolink II & 10 & 0 & 0 \\
Laser-ablated/Maxcem & 3 & 7 & 0 \\
\hline
\end{tabular}

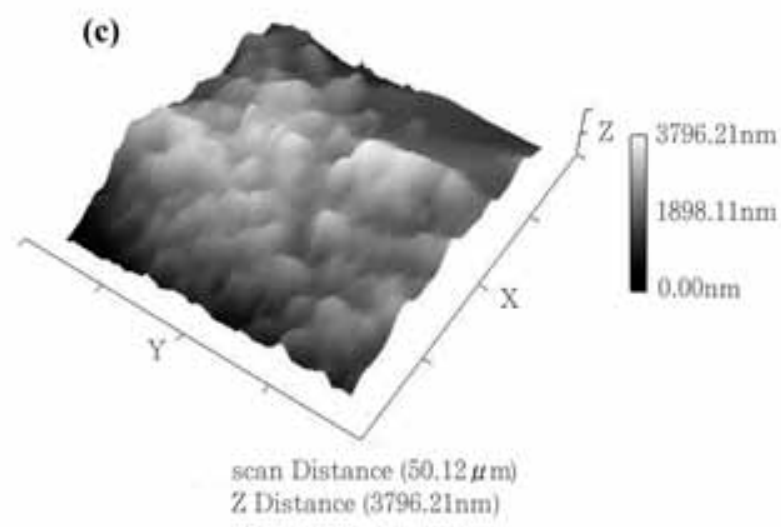

(d)

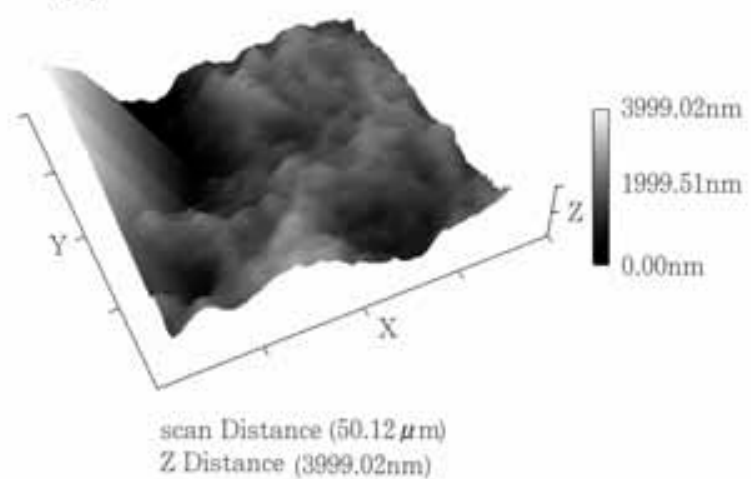

Fig. 4 Representative images of dentin disk surfaces after receiving (a) diamond bur preparation plus acid etching, (b) diamond bur preparation only, (c) laser preparation plus acid etching, and (d) laser preparation only. Striations (arrow) could be found in the dentin surface prepared by diamond bur only (b). Laser-irradiated surface (d) exhibited the greatest surface irregularity. 
the cavity was prepared by Er, Cr: YSGG laser and cemented with Maxcem (Fig. 2(d)).

Tensile bond strength measurement and failure mode analysis

The means and standard deviations of the tensile bond strengths of bur-cut/Variolink II, burcut/Maxcem, laser-ablated/Variolink II, and laserablated/Maxcem were $19.11 \pm 5.88 \mathrm{MPa}, 8.54 \pm 2.38$ $\mathrm{MPa}, 13.72 \pm 3.43 \mathrm{MPa}$, and $12.11 \pm 3.71 \mathrm{MPa}$, respectively (Fig. 3). No statistically significant differences were found between laser-ablated/Variolink II and laser-ablated/Maxcem, as well as between burcut/Maxcem and laser-ablated/Maxcem.

The predominant fracture modes of burcut/Variolink II, bur-cut/Maxcem, laser-ablated/ Variolink II, and laser-ablated/Maxcem were Type 1 (70\%), Type $1(100 \%)$, Type $1(100 \%)$, and Type 2 (70\%), respectively (Table 2 ).

Surface roughness analysis of dentin disks by AFM Figure 4 shows the representative images of dentin disk surfaces after receiving different treatments. The surface that received diamond bur preparation plus acid etching (Fig. 4(a)) exhibited 253.28 16.79 $\mathrm{nm}$ of surface roughness (Fig. 5). Striations could be found in the dentin surface prepared by diamond bur only (Fig. 4(b)). In addition, this group exhibited the

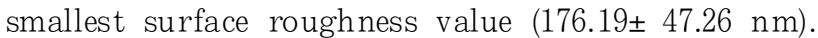
When the surface was treated by laser irradiation and acid etching (Fig. 4(c)), the surface roughness $(620.30 \pm 80.77 \mathrm{~nm})$ was greater than those of previous two groups. Finally, laser irradiated-only surface (Fig. 4(d)) exhibited the greatest surface irregularity

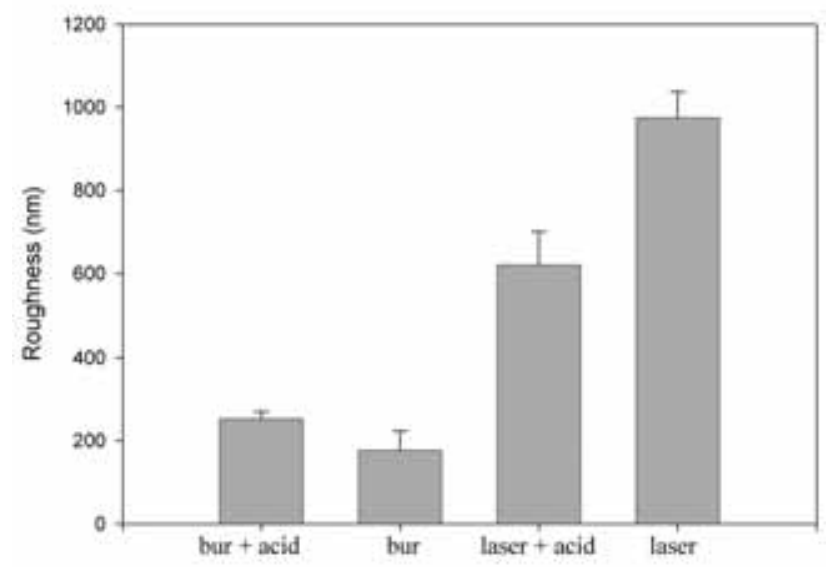

Fig. 5 Means and standard deviations of surface roughness of dentin specimens after receiving diamond bur preparation plus acid etching, diamond bur preparation only, laser preparation plus acid etching, and laser preparation only were $253.28 \pm$ $16.79 \mathrm{~nm}, 176.19 \pm 47.26 \mathrm{~nm}, 620.30 \pm 80.77 \mathrm{~nm}$, and $975.00 \pm 62.90 \mathrm{~nm}$, respectively. There were significant differences between any two groups $(n=15)$. and surface roughness value $(975.00 \pm 62.90 \mathrm{~nm})$. Further, as shown in Fig. 5, significant differences existed between any two groups.

\section{DISCUSSION}

Currently, the most widely accepted concept of dentin bonding is based on the formation of a hybrid layer. The underlying mechanism is that acidic conditioners dissolve the smear layer and demineralize the hydroxyapatite crystals of dentin substrate, followed by infiltration of monomers into the exposed collagen fiber $\mathrm{s}^{15)}$. The bonding mechanism of commercial products developed using this concept can be grossly divided into two types: total-etch versus self-etch luting materials. Total-etch luting materials are characterized by a complete removal of the smear layer with $37 \%$ phosphoric acid, while a self-etch luting material combines the etchant and primer into one application to achieve simultaneous demineralization and resin infiltration ${ }^{16}$.

In this study, Variolink II and Maxcem were chosen as representative resin cements of total-etch and self-etch, one-step luting materials, respectively. Both products were designed for cementation of crowns, inlays, and veneers - especially those made of composite or porcelain. Due to increasing esthetic demand in the restoration of large-sized posterior cavities, composite inlays (belleGlass HP) were used as the restorative material instead of gold inlays.

The bond strength at dentin-resin cement interface depends on a number of factors: hybrid layer thickness, resin tag length, adhesive lateral branch formation, surface roughness, interface integrity, and void formation ${ }^{17}$. When the cavity was prepared by a bur and then etched with phosphoric acid for 30 seconds, no debris or smear layer was left (Fig. 1(a)). Moreover, peritubular and intertubular dentin were excessively demineralized, resulting in enlarged dentinal tubule orifices. The topography under AFM examination (Fig. 4(a)) revealed a relatively flat surface compared with the laser-irradiated surfaces (Figs. 4(c) and (d)). Surface roughness value was $253.28 \pm 16.79 \mathrm{~nm}$, which was significantly smaller than those of lased surfaces (Fig. 5).

However, after demineralization in $6 \mathrm{~N} \mathrm{HCl}$ and deproteinization in $1 \% \mathrm{NaOCl}$, dentin-resin cement interface of the bur-cut/Variolink II group revealed many uniformly distributed resin tags of $50 \mu \mathrm{m}$ length (Fig. 2(a)). These resin tags aggregated initially and subsequently diverted into dentinal tubules. The aggregation was probably derived from the hybrid layer formation which represented the entanglement of resin monomers with collagen framework. Excite DSC, a dual-polymerized onebottle dentin adhesive used in this group, has been demonstrated to form a hybrid layer, resin tags, and 
adhesive lateral branches ${ }^{18}$. Its efficacy was attributed to inclusion of nanoscale inorganic fillers (Table $1)$, which could carry the primer-adhesive solution into a deeper area that could not be reached by ligh $\mathrm{t}^{19}$. As hybrid layer principally contributed to dentin-material bond strength, the well-formed hybrid layer, resin tags, and adhesive lateral branches of this group resulted in the greatest bond strength (19.11 $\pm 5.88 \mathrm{MPa})$ (Fig. 3). Furthermore, fractured surfaces of two debonded specimens revealed complete adhesive failure between resin cement and composite inlay (Table 2), indicating that the bond strength of dentin-resin cement was occasionally even greater than that of resin cementcomposite inlay.

When the cavity was prepared by a high-speed diamond bur without subsequent acid etching, the surface should be covered with an amorphous smear layer. The AFM image showed striations on the smear layer which were left by diamond bur cutting (Fig. 4(b)). Smear layer covering also reduced the surface roughness, consequently causing this group (but-cut/Maxcem) to exhibit the lowest surface roughness value $(176.19 \pm 47.26 \mathrm{~nm})$ (Fig. 5). In addition, the dentin-resin cement interface of burcut/Maxcem group did not display any resin tags (Fig. 2(b)). The reason suspected was that the weak acidity of one-step, self-etching resin cement could not entirely dissolve the thick smear layer and smear plugs, thereby preventing the acidic monomers from penetrating into the dentin matrix ${ }^{20)}$. Therefore, this group exhibited the smallest tensile bond strength value $(8.54 \pm 2.38 \mathrm{MPa})$ (Fig. 3), which was significantly lower than those of bur-cut/Variolink II and laser-ablated/Variolink II groups. Moreover, all debonded specimens demonstrated complete adhesive failure between resin cement and dentin (Table 2).

The Er, Cr: YSGG laser with 2,780 $\mathrm{nm}$ of wavelength is highly absorbed by water and hydroxyapatite. After efficient absorption of laser energy, water droplets produce violent microexpansion and simultaneously cut dental hard tissues ${ }^{21)}$. In comparison with high-speed bur preparation, cavities prepared with laser are relatively painless and produce less vibration and heat ${ }^{22)}$. Another advantage of laser preparation that has been mentioned is that the irradiated surface is more acid-resistant. This is because laser can reduce the carbonate-to-phosphate ratio, produce less acidsoluble compounds, and consequently enhance the resistance to acid demineralization ${ }^{23)}$.

Bond strengths of porcelain laminate veneers to tooth surfaces etched with acid or Er, Cr: YSGG laser have been compared ${ }^{10)}$. Results showed no differences in microtensile bond strength between these two etching methods. The pulpal temperature elevation induced by Er, Cr: YSGG laser has been reported to be $2{ }^{\circ} \mathrm{C}^{8)}$, and no pulpal inflammatory responses were triggered ${ }^{9}$. However, temperature increase of $2.8^{\circ} \mathrm{C}$ at interodontoblastic vacuoles just below the preparation was noted ${ }^{24)}$. In addition, significantly enhanced synthesis of inflammatory mediator, leukotriene B4, in human pulp cells was observed following thermal stimulation at $38^{\circ} \mathrm{C}^{25}$. At this juncture, it is expedient to highlight and caution about the issue of temperature rise during laser irradiation, even though the usage safety of laser treatment in the clinic is widely accepted.

In this study, the lased dentin surface displayed a scaly, irregular, and rugged appearance (Fig. 1(b)). No smear layer or debris was noted, and open dentinal tubule orifices were also not enlarged. In addition, the laser ablated more organic portion than the inorganic composition, causing peritubular dentin to protrude from the surrounding intertubular dentin. This effect was contrary to that of acid etchant, which preferentially etched peritubular dentin. The AFM image of laser-irradiated surface (Fig. 4(d)) showed high irregularities and yielded the greatest surface roughness value $(975.00 \pm 62.90 \mathrm{~nm})$ (Fig. 5).

After acid etching, the protruded peritubular dentin was flattened (Figs. 1(c) and 4(c)), thus reducing the surface roughness value to $620.30 \pm$ $80.77 \mathrm{~nm}$ (Fig. 5). However, it was still significantly greater than the surface roughness of bur preparation either with or without acid etching.

With the laser-ablated/Maxcem group, no resin tag formation and observable hybrid layer could be found at the dentin-resin cement interface (Fig. 2(d)). This was due to the weak acidity of Maxcem as described previously. Although laser-ablated/ Maxcem group exhibited a greater surface roughness value, and that greater presence of peritubular dentin has been suggested to achieve better bonding to dentin ${ }^{22}$, this group exhibited a mere tensile bond strength of $12.11 \pm 3.71 \mathrm{MPa}$ - which was not significantly greater than that of bur-cut/Maxcem group (Fig. 3). One possible explanation was that laser irradiation could cause decomposition of collagen fibers, as characterized by two absorption bands at 2200 and $2015 \mathrm{~cm}^{-1}$ in Fourier transform infrared analysis ${ }^{26)}$. Against this background, hybrid layer was not well formed. The predominant fracture mode was partial adhesive failure between resin cement and dentin, and partial adhesive failure between resin cement and composite inlay, indicating that Maxcem bonded roughly equally to both dentin and composite inlay.

The dentin-resin cement interface of laserablated/Variolink II group (Fig. 2(c)) revealed some resin tags with length longer than that of burcut/Variolink II group (Fig. 2(a)). However, these resin tags did not conglomerate and distributed 
relatively scarcely. In addition, possible decomposition of collagen fibers ${ }^{26)}$ after laser irradiation might jeopardize hybrid layer formation. Consequently, the tensile bond strength $(13.72 \pm 3.43 \mathrm{MPa})$ of laserablated/Variolink II group was significantly lower than that of bur-cut/Variolink II group, but significantly higher than that of bur-cut/Maxcem group (Fig. 3).

Although the surface roughness of dentin is not a critical factor to enamel bonding, the influence of surface roughness of laser-irradiated dentin on bond strength has not been evaluated. From the results of this study, it could be seen that when Variolink II was used, the bond strength of laser-ablated specimens was lower than that of bur-cut specimens even though the former exhibited a greater surface roughness than the latter. Moreover, the tensile bond strength of total-etch resin cement was greater than that of self-etch, one-step resin cement, irrespective of whether the cavity was prepared by bur or Er, Cr: YSGG laser. It should be noted that the adhesive bonding of luting cements is closely related to the fracture resistance of restoration $\mathrm{s}^{277}$.

Nevertheless, self-etch resin cements are less technique-sensitive and present less postoperative sensitivity $^{28}$. Therefore, the use of total-etch or selfetch resin cement depends on the clinical situation and the operator's judgment. It should also be put into perspective that the results of in vitro tests cannot be directly applied to clinical conditions. Ultimately, the final performance of a material should be established in a long-term clinical study.

\section{CONCLUSION}

Er, Cr: YSGG laser irradiation adversely affected the adhesion of composite inlays to dentin with Variolink II as the resin cement. Compared to Maxcem, Variolink II was found to produce higher tensile bond strengths of composite inlays to dentin.

\section{REFERENCES}

1) Stern, RH, Sognnaes, RF. Laser beam effect on dental hard tissues. J Dent Res 1964; 43: 873.

2) Goldman L, Gray JA, Goldman J, Goldman B, Meyer R. Effects of laser beam impacts on teeth. J Am Dent Assoc 1965; 70: 601-606.

3) Harashima T, Kinoshita J, Kimura Y, Brugnera A, Zanin F, Pecora JD, Matsumoto K. Morphological comparative study on ablation of dental hard tissues at cavity preparation by Er: YAG and Er, Cr: YSGG lasers. Photomed Laser Surg 2005; 23: 52-55.

4) Kinoshita J, Kimura Y, Matsumoto K. Comparative study of carious dentin removal by Er, Cr: YSGG laser and Carisolv. J Clin Laser Med Surg 2003; 21: 307-315.

5) Hossain M, Nakamura Y, Tamaki Y, Yamada Y, Murakami Y, Matsumoto K. Atomic analysis and knoop hardness measurement of the cavity floor prepared by Er, Cr: YSGG laser irradiation in vitro. J Oral Rehabil 2003; 30: 515-521.

6) Schoop U, Kluger W, Moritz A, Nedjelik N, Georgopoulos A, Sperr W. Bactericidal effect of different laser systems in the deep layers of dentin. Lasers Surg Med 2004; 35: 111-116.

7) Gutknecht N, Apel C, Schäfer C, Lampert F. Microleakage of composite fillings in Er, Cr: YSGG laser-prepared class II cavities. Lasers Surg Med 2001; 28: 371-374.

8) Rizoiu I, Kohanghadosh F, Kimmel AI, Eversole LR. Pulpal thermal responses to an erbium, chromium: YSGG pulsed laser hydrokinetic system. Oral Surg Oral Med Oral Pathol Oral Radiol Endod 1998; 86: 220-223.

9) Eversole LR, Rizoiu I, Kimmel AI. Pulpal response to cavity preparation by an erbium, chromium: YSGG laser-powered hydrokinetic system. J Am Dent Assoc 1997; 128: 1099-1106.

10) Usumez A, Aykent F. Bond strengths of porcelain laminate veneers to tooth surfaces prepared with acid and Er, Cr: YSGG laser etching. J Prosthet Dent 2003; 90: 24-30.

11) Mak YF, Lai SCN, Cheung GSP, Chan AWK, Tay FR, Pashley DH. Micro-tensile bond testing of resin cements to dentin and an indirect resin composite. Dent Mater 2002; 18: 609-621.

12) Trajtenberg CP, Pereira PNR, Powers JM. Resin bond strength and micromorphology of human teeth prepared with an erbium: YAG laser. Am J Dent 2004; 17: 331-336.

13) Goracci C, Fabianelli A, Sadek FT, Papacchini F, Tay FR, Ferrari M. The contribution of friction to the dislocation resistance of bonded fiber posts. J Endodon 2005; 31: 608-612.

14) Lee BS, Lin PY, Chen $\mathrm{MH}$, Hsieh $\mathrm{TT}$, Lin $\mathrm{CP}$, Lai JY, Lan WH. Tensile bond strength of Er, Cr: YSGG laser-irradiated human dentin and analysis of dentin-resin interface. Dent Mater 2007; 23: 570-578.

15) Nakabayashi N, Kojima K, Masuhara E. The promotion of adhesion by the infiltration of monomers into tooth substrates. J Biomed Mater Res 1982; 16: 265273.

16) Bishara SE, Oonsombat C, Ajlouni R, Laffoon JF. Comparison of the shear bond strength of 2 self-etch primer adhesive systems. Am J Orthod Dentofacial Or thop 2004; 124: 348-350.

17) Roulet JF, Degrange M. Adhesion: The silent revolution. J Adhes Dent 1999; 3: 285-287.

18) Dagostin A, Ferrari M. Effect of dual bonding technique on the bond strength of ceramic restorations. Dent Mater 2002; 18: 304-310.

19) Ferrari M, Vichi A, Grandini S, Goracci C. Efficacy of a self-curing adhesive/resin cement system on luting Vectris fiber posts into root canals: a SEM investigation. Int J Prosthed 2001; 14: 543-549.

20) Ogata M, Harada N, Yamaguchi S, Nakajima M, Pereira PN, Tagami J. Effects of different burs on dentin bond strengths of self-etching primer bonding systems. Oper Dent 2001; 26: 375-382.

21) Fried D, Ashouri N, Breunig T, Shori R. Mechanism of water augmentation during IR laser ablation of dental enamel. Lasers Surg Med 2002; 31: 186-193.

22) Visuri SR, Gilbert JL, Wright DD, Wigdor HA, Walsh JT Jr. Shear strength of composite bonded to 
Er: YAG laser-prepared dentin. J Dent Res 1996; 75: 599-605.

23) Martinez-Insua A, Da Silva Dominguez L, Rivera FG, Santana-Penin UA. Differences in bonding to acidetched or Er: YAG-laser-treated enamel and dentin surfaces. J Prosthet Dent 2000; 84: 280-288.

24) Zach L, Cohen G. Pulp response to externally applied heat. Oral Surg Oral Med Oral Path 1965; 19: 515530 .

25) Eberhard J, Zahl A, Dommisch H, Winter J, Acil Y, Jepsen S. Heat shock induces the synthesis of the inflammatory mediator leukotriene B4 in human pulp cells. Int Endod J 2005; 38: 882-888.

26) Lee BS, Lin CP, Hung YL, Lan WH. Structural changes of Er: YAG laser-irradiated human dentin. Photomed Laser Surg 2004; 22: 330-334.

27) Furukawa K, Inai N, Tagami J. The effects of luting resin bond to dentin on the strength of dentin supported by indirect resin composite. Dent Mater 2002; 18: 136-142.

28) Sensat ML, Brackett WW, Meinberg TA, Beatty MW. Clinical evaluation of two adhesive composite cements for the suppression of dentinal cold sensitivity. J Prosthet Dent 2002; 88: 50-53. 